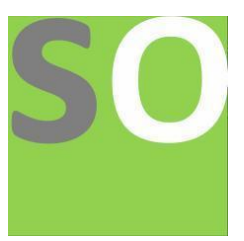

Article title: Study of gravitational constant and mercury's precession from perspective of physical aesthetics and ideal fluid

Authors: Yun-Gang Li[1], Cheng-Shu Li[2]

Affiliations: Hainan Shulin Education Group Co., Ltd.[1], Hefei University of Technology[2]

Orcid ids: 0000-0003-1382-8467[1], 0000-0002-2651-7214[2]

Contact e-mail: 576429680@qq.com

License information: This work has been published open access under Creative Commons Attribution License http://creativecommons.org/licenses/by/4.0/, which permits unrestricted use, distribution, and reproduction in any medium, provided the original work is properly cited. Conditions, terms of use and publishing policy can be found at https://www.scienceopen.com/.

Preprint statement: This article is a preprint and has not been peer-reviewed, under consideration and submitted to ScienceOpen Preprints for open peer review.

DOI: 10.14293/S2199-1006.1.SOR-.PPZPJHE.v1

Preprint first posted online: 12 May 2020

Keywords: gravitational constant, derived constant, physical aesthetics, perihelion precession, Inverse-Square, general relativity 


\title{
Study of gravitational constant and mercury's precession from perspective of physical aesthetics and ideal fluid
}

\author{
Yun-Gang $\mathrm{Li}^{1 *} \&$ Cheng-Shu $\mathrm{Li}^{2}$
}

\begin{abstract}
${ }^{1}$ Hainan Shulin Education Group Co., Ltd., Haikou 570226, China. ${ }^{2}$ Hefei University of Technology, Xuancheng 242000, China. *e-mail:576429680@qq.com, mobile:+8613876908506
\end{abstract}

\begin{abstract}
Newton's law of universal gravitation does not explain the abnormal precession of Mercury, and the values of gravitational constant $G$ measured by different research teams does not coincide. This paper had study of these two problems from perspective of physical aesthetics and ideal fluid and derived a new formula for calculating the exact $G$ value by using the speed of light in a vacuum, which including error correction formulas and verified by experimental data of other scientists. After corrected, the $G$ values measured by some famous surveyors approximately coincides with a specific value. The formula also verified by the observation data of the abnormal precession of the perihelion of mercury, it contributes 35.94"/cy of additional observations to the abnormal precession of Mercury, which is only about 2 "/cy different from the observed value of Le Verrier, this give another explanation for the abnormal precession of Mercury which is completely different from Einstein's general theory of relativity. The preliminary Conclusions is that G equals $1 /(16 \pi c)$. For further verify the formulas, this paper also designed an experimental plan for the space agency to disprove Einstein's GTR.
\end{abstract}

Keywords: gravitational constant, derived constant, physical aesthetics, perihelion precession, Inverse-Square, general relativity PACS: 04.80.Cc, 06.20.Jr, 95.30.Sf, 96.30.Dz

\section{INTRODUCTION}

The abnormal precession of Mercury's perihelion equals $43 \mathrm{H} / \mathrm{cy}$ is the core proof to prove that Einstein's general relativity is right. Newton's law of universal gravitation does not explain the abnormal precession of Mercury's perihelion, and the gravitational constant $\mathrm{G}$ values measured by different research teams in the world do not coincide with each other within the error range for more than 200 years, for example, the difference between the results of BIPM-14 and LENS-14 collected by CODATA-2014 is as high as $544 \mathrm{ppm}^{1}$, this may cause by undiscovered systematic errors.

In 2018, Luo Jun, an academician of the Chinese Academy of Sciences, led his team and measured the highest precise gravitational constant $\mathrm{G}$ in the world, including seven values by TOS method ${ }^{2,3}$. We studied their experimental data and the experimental data of Cavendish in $1798^{4}$, Luther in $1982^{5,6}$, Boys in $1895^{15}$, and many other research teams, and got a formula for calculating the exact value of the gravitational constant $\mathrm{G}$ using the speed of light in a vacuum, including the error correction formulas between the theoretical exact $G$ value and the measured $G$ value, then the measured $G$ values approximately coincides with $1 /(16 \pi \mathrm{c})$. The correction formulas also verified by the observation data of the abnormal precession of mercury, it is another explanation for the abnormal precession of Mercury which is completely different from Einstein's general relativity.

\section{METHOD}

Physics is beautiful, it contains several aspects, such as simple, symmetry, harmonious and unity beauty, we explore Newton's law of universal gravitation and the gravitational constant $\mathrm{G}$ by using physical aesthetics, and treat gravitational field as flow field of ideal fluid. Formula(1) is the mathematical expression of Newton's law of universal gravitation, and has the characteristics of physical beauty.

$$
F=\mathrm{G} \frac{M m}{r^{2}}
$$

Where $\mathrm{G}$ is the gravitational constant. The recommended $\mathrm{G}$ value (CODATA-2014) is $6.67408 \mathrm{E}-11 \mathrm{~N} \cdot \mathrm{m}^{2} / \mathrm{kg}^{2}{ }^{1}$. We found that the product of $\mathrm{G}$ and the speed of light in vacuum $\mathrm{c}$ $\left(299792458 \mathrm{~m} / \mathrm{s}^{1}\right)$ is equal to $0.020008388, \mathrm{G}$ is very close to $1 /(50 \mathrm{c})$, the difference is only $0.419 \%$.

$$
F \approx \frac{1}{50 c} \frac{M m}{r^{2}}
$$

The proportional factor in Eq.(2) contains an integer 50, which has simple characteristics, but lacks beauty, 50, 1/50 and $1 /(50 \mathrm{c})$ have no special physical meaning, so $\mathrm{G}=1 /(50 \mathrm{c})$ is, therefore, an approximation, a coincidence, but not the truth.

Formula (3) is the expression of Coulomb's law:

$$
F=\mathrm{k} \frac{q_{1} q_{2}}{r^{2}}=\frac{1}{4 \pi \varepsilon_{0}} \frac{q_{1} q_{2}}{r^{2}}=\frac{1}{\varepsilon_{0}} \frac{q_{1} q_{2}}{4 \pi r^{2}}
$$

Where $4 \pi r^{2}$ is the spherical area of a virtual sphere which radius is equal the distance of the two point charges and one of the point charges is the sphere center. By analogy with Coulomb's law, we add spherical area $4 \pi \mathrm{r}^{2}$ into Newton's gravitational formula, and reserved $\mathrm{c}$ (The speed of light in vacuum, which was added in Eq.(2)), the gravitational formula becomes:

$$
F=\mathrm{G} \frac{M m}{r^{2}}=\frac{1}{K_{1} c} \frac{M m}{4 \pi r^{2}}
$$

Where $K_{1}=\frac{1}{4 \pi c \mathrm{G}} \approx 3.9772 \approx 4$

The integer one, two and four are the most common numbers with simple beauty in many physical and mathematical formulas, so it can be inferred that $\mathrm{K}_{1}$ is equal to integer four, but the denominator of Eq.(4) already contains $4 \pi \mathrm{r}^{2}$, if the 
denominator contains another integer four, it obviously does not have physical meaning, therefore, calculate the square root for integer four and get two integer two, and add to Eq.(4), then:

$$
F=\frac{K_{2}}{c} \frac{\frac{M}{2} \frac{m}{2}}{4 \pi r^{2}}
$$

We can obtain $\mathrm{K}_{2}=16 \pi \mathrm{cG}=1.005731 \approx 1$ from Eq.(1),(5). If $\mathrm{K}_{2}$ equal to integer one, the proportional coefficient is only related to the speed of light in vacuum, and becomes extremely simple and has special physical significance, then Eq.(5) becomes another equation with simple beauty:

$$
F=\frac{1}{c} \frac{\frac{M}{2} \frac{m}{2}}{4 \pi r^{2}}
$$

From Eq.(1),(6):

$$
\mathrm{G}=\frac{1}{16 \pi c}
$$

The constant one in Eq.(6),(7) is containing units $\mathrm{Wm}^{2} / \mathrm{kg}^{2}$. We use $\mathrm{P}$ to represent this constant and move $\mathrm{c}$ to the left, then:

$$
F C=P \frac{\frac{M}{2} \frac{m}{2}}{4 \pi r^{2}}
$$

When a physical equation has the characteristics of physical beauty, and each variables and coefficients had a specific physical meaning, it may close to the truth. For Eq.(8), the left side $\mathrm{Fc}$ is the expression of power, and the right side, $\mathrm{P}=1 \mathrm{~W} \cdot \mathrm{m}^{2} / \mathrm{kg}^{2}$, it has the most simple beauty, so we inferred that Eq. (6) $\sim(8)$ is close to the truth.

In order to explain this, we put forward a new theory called half-effective-field theory: In the gravitational field of two particles of $\mathrm{M}$ and $\mathrm{m}$, only the half gravitational field that near the $\mathrm{m}$ side of the particle $\mathrm{M}$ has an influence on the particle $\mathrm{m}$, and for the particle $\mathrm{m}$, only the half gravitational field that near the $\mathrm{M}$ side of the particle $\mathrm{m}$ has an influence on the particle $\mathrm{M}$.

Eq.(7) can be used for calculating the theoretical gravitational constant $\mathrm{G}$ when the mass $\mathrm{M}$ and $\mathrm{m}$ are particles, but we obtain that $\mathrm{G}=6.6360468 \mathrm{E}-11 \mathrm{~N} \cdot \mathrm{m}^{2} / \mathrm{kg}^{2}$, there is still a large deviation from the CODATA-2014 recommended value $6.67408 \mathrm{E}-11 \mathrm{~N} \cdot \mathrm{m}^{2} / \mathrm{kg}^{2}$, there may be some system errors not considered in the measurement of $\mathrm{G}$ value, so the measured $\mathrm{G}$ value needs to be corrected.

In general, the correct condition of formula (1) is that the mass $\mathrm{M}$ and $\mathrm{m}$ must be particles. However, it is found that the mass of uniform spherical shell or uniform sphere can be regarded as particle by integral calculation, this is why all the research teams treat the uniform sphere as a particle and do not correct the measured value of $\mathrm{G}$.

If the gravitational field is regard as an ideal fluid, and the gravitational field around the mass is regard as a point sink, gravitation is the difference between the forces on both sides of the mass, one tangible sphere is bound to block part of the flow field (gravitational field) of another mass, and resulting increase the gravitation. The gravitational correction coefficient is proportional to the geometric average value of blocking area of the two mass and inversely proportional to the surface area $S_{r}$ of the virtual sphere, where $S_{r}=4 \pi r^{2}, r$ is the centroids distance of the two masses.

$$
\begin{aligned}
& \mathrm{F}=(1+K) G \frac{M m}{r^{2}} \\
& K=\frac{\sqrt{S_{M} S_{m}}}{S_{r}}=\sqrt{\frac{S_{M}}{s_{r}}} \sqrt{\frac{S_{m}}{S_{r}}}=K_{M} K_{m}
\end{aligned}
$$

Where $\mathrm{K}_{\mathrm{M}}$ is the correction coefficient of attractive mass $\mathrm{M}$, $\mathrm{K}_{\mathrm{m}}$ is the correction coefficient for measuring mass $\mathrm{m}, \mathrm{S}_{M}$ is the projection area when $M$ is projected to a virtual sphere with $r$ as the radius, taking the centroid of $\mathrm{m}$ as the projection center, $\mathrm{S}_{\mathrm{m}}$ is the projection area when $\mathrm{m}$ is projected to a virtual sphere with $r$ as the radius, taking the centroid of $\mathrm{M}$ as the projection center.

When the mass $M$ and $m$ are spheres:

$$
\begin{aligned}
& S_{M}=2 \pi r^{2}\left(1-\sqrt{1-\frac{R_{M}^{2}}{r^{2}}}\right) \\
& S_{m}=2 \pi r^{2}\left(1-\sqrt{1-\frac{R_{m}^{2}}{r^{2}}}\right)
\end{aligned}
$$

When $r>R_{m}$ and $r>>R_{M}$, Eq.(10) can approximately become Eq.(13) and has simple beauty with the same form as formula(1):

$$
K=\frac{\sqrt{S_{M} S_{m}}}{S_{r}} \approx \frac{R_{M} R_{m}}{4 r^{2}}
$$

Furthermore, we think that the essence of blocking is blocking mass flux, so:

$$
K_{m}=\sqrt{\frac{M_{b}}{M}}
$$

Where $\mathrm{M}_{\mathrm{b}}=\mathrm{MS}_{\mathrm{m}} / \mathrm{S}_{\mathrm{r}}$ and the maximum $\mathrm{M}_{\mathrm{b}}$ should not exceed $\mathrm{m} / 2$, and then we obtain more inclusive equations:

$$
\begin{aligned}
& K_{M}=\left(\frac{S_{M}}{S_{r}}\right)^{a} \\
& K_{m}=\left\{\begin{array}{l}
\left(\frac{S_{m}}{S_{r}}\right)^{a}, \frac{m}{2}>\frac{S_{m}}{S_{r}} M \\
\left(\frac{m}{2 M}\right)^{a}, \frac{m}{2} \leq \frac{S_{m}}{S_{r}} M
\end{array}\right.
\end{aligned}
$$

The $\mathrm{m} / 2$ in Eq.(16) shows that the half-effective-field theory is self-consistency. Exponent a is related to the shape of the mass, when the mass is spherical, the exponent $a=0.5$, for cuboid, $\mathrm{a}=1$.

Finally, the $\mathrm{G}$ value can be calculate from the measured value $\mathrm{G}_{\text {measured }}$ by Eq.(17):

$$
\mathrm{G}=\frac{\mathrm{G}_{\text {measured }}}{(1+\mathrm{K})}
$$

\section{EXPERIMENTAL RESULT}

We verified our theory by the measured data of other scientists in two aspects, the measurement of $G$ and the abnormal precession of Mercury.

\section{GRAVITATIONAL CONSTANT G}

At present, it is recognized that the $G$ value measured by Cavendish is $6.754 E-11 \mathrm{~N} \cdot \mathrm{m}^{2} / \mathrm{kg}^{2}$, this $\mathrm{G}$ value can be calculate from the density of the earth he measured, the dimensions selected in the experiment with torsion balance of Cavendish are as follows: Large mass(ball) $\mathrm{M}$ is 12 inches in diameter, small mass(ball) $\mathrm{m}$ is 2 inches in diameter, the centroid distance of the large ball and the small ball is 8.85 inches ${ }^{4}$, the selected dimensions meets the condition $\mathrm{m} / 2<\mathrm{MS}_{\mathrm{m}} / \mathrm{S}_{\mathrm{r}}$, and the second term of Eq.(16) should be selected for calculating $\mathrm{K}$, then $\mathrm{K}=$ 0.017504 , the measured $\mathrm{G}$ value is corrected to $6.6378 E-11$ $\mathrm{N} \cdot \mathrm{m}^{2} / \mathrm{kg}^{2}$, the error between $6.6378 E-11$ and $1 /(16 \pi \mathrm{c})$ is 264 
ppm, and it's unbelievably precise in 1798 .

In 1982, Luther's experiment, the measured $G$ value is $6.6726 E-11 \mathrm{~N} \cdot \mathrm{m}^{2} / \mathrm{kg}^{2}$, the large mass $\mathrm{M}$ are two spheres with an average diameter of $101.6509 \mathrm{~mm}$, the centroid distance between the large mass is $140.59454 \mathrm{~mm}$, the two small mass $\mathrm{m}$ are disks with diameter of $7.166 \mathrm{~mm}$, and mounted on a hollow tube, rod $28.5472 \mathrm{~mm}$ long, the mass of the two disks is $5 \mathrm{~g} 5$. Although the small mass is different from the sphere, but because the disks very thin and has little influence in other direction, we choose 0.5 for exponent a just like spheres. The centroid distance between $\mathrm{M}$ and $\mathrm{m}$ is $59.60667 \mathrm{~mm}$, and the correction coefficient $\mathrm{K}=0.005334$, the corrected $\mathrm{G}$ value is $6.6372 E-11 \mathrm{~N} \cdot \mathrm{m}^{2} / \mathrm{kg}^{2}$, the error between $6.6372 E-11$ and $1 /(16 \pi \mathrm{c})$ is $173 \mathrm{ppm}$.

In 2018, Luo Jun, an academician of the Chinese Academy of Sciences, measured the highest precision gravitational constant in the world at present, include seven values by TOS method. Because Luo Jun's team adopted the periodic method, the pendulum is a cuboid about $90 \mathrm{~mm}$ long ${ }^{2,3}$, so the pendulum need to be treated as two connected small mass $\mathrm{m}$. And because the part of gravitation that needs to be corrected is proportional to the reciprocal of the quartic power of $r$, therefore, the shortrange configuration only needs to consider the gravitational correction between the small mass $\mathrm{m}$ and the large mass $\mathrm{M}$, the influence of another large mass $\mathrm{M}$ can be ignored. For remote configuration, at the equilibrium position, the gravitation between the two small masses $m$ and each large mass $M$ is almost the same, so for remote configurations, the effect of placing the two large balls $\mathrm{M}$ is almost same as not placing the two large balls, when the torsional angle of the cuboid pendulum is very small, therefore, the correction of gravitation can be ignored. And because the pendulum is a cuboid, so exponent $\mathrm{a}=1$. We try to calculate the correction coefficient $\mathrm{K}_{\mathrm{m}}$ :

$$
\mathrm{K}_{m}=\frac{S_{m}}{S_{r}}=\frac{1}{\pi} \times \arctan \left(\frac{W}{2 r-L}\right) \times \arctan \left(\frac{H}{2 r-L}\right)
$$

Where $\mathrm{m}$ is a half of the cuboid pendulum, and the center of mass $m$ is located at $1 / 4$ length of the pendulum. $S_{m}$ is the projection area when the near half pendulum $(\mathrm{m})$ is projected to the virtual sphere with $r$ as the radius with the center of mass of $\mathrm{M}$ as the projection center. $\mathrm{W}$ is the width of the pendulum, $\mathrm{H}$ is the height of the pendulum, $\mathrm{L}$ is half the length of the pendulum(That is, the length of $\mathrm{m}$ ). But it also meets the condition $\mathrm{m} / 2<\mathrm{MS}_{\mathrm{m}} / \mathrm{S}_{\mathrm{r}}$, so, the second term of Eq.(16) should be selected, then we obtain the corrected $\mathrm{G}$ values and the errors between the theoretical value $6.6360468 \mathrm{E}-11 \mathrm{~N} \cdot \mathrm{m}^{2} / \mathrm{kg}^{2}$.

Table1 Corrected G value of Luo Jun and errors (2018)

\begin{tabular}{|c|c|c|c|c|c|}
\hline $\begin{array}{c}\text { Experim } \\
\text { ent } \\
\end{array}$ & $\begin{array}{l}\text { Measured }{ }^{[2,3]} \\
10^{-11} \mathrm{Nm}^{2} / \mathrm{kg}^{2}\end{array}$ & $\begin{array}{c}\text { Corrected } \\
10^{-11} \mathrm{Nm}^{2} / \mathrm{kg}^{2} \\
\end{array}$ & $\begin{array}{l}\text { error } \\
\mathrm{ppm}\end{array}$ & \multicolumn{2}{|c|}{$\begin{array}{c}\text { weighted mean values } \\
10^{-11} \mathrm{Nm}^{2} / \mathrm{kg}^{2}\end{array}$} \\
\hline G1 & 6.674154 & 6.635627 & -63 & 6.635661 & \multirow{5}{*}{$\begin{array}{c}6.635704 \\
\text { TOS-I }\end{array}$} \\
\hline G2 & 6.674222 & 6.635695 & -53 & Fibre1 & \\
\hline G3 & 6.674237 & 6.635710 & -51 & $\begin{array}{l}6.635710 \\
\text { Fibre2 }\end{array}$ & \\
\hline G4 & 6.674274 & 6.635747 & -45 & 6.635743 & \\
\hline G5 & 6.674266 & 6.635739 & -46 & Fibre3 & \\
\hline G6 & 6.674017 & 6.636464 & 63 & 6.636508 & 6.636508 \\
\hline G7 & 6.674105 & 6.636552 & 76 & Fibre4 & TOS-II \\
\hline iverage & 6.674182 & 6.635933 & -17 & & 6.636106 \\
\hline
\end{tabular}

Table1 shows the simple average of the corrected G values is 6.635933 with error $-17 \mathrm{ppm}$, and the weighted mean value is 6.636106 with error $9 \mathrm{ppm}$.

\section{ABNORMAL PRECESSION OF THE PLANETS}

Eq.(19) is the differential equation of motion of the planets, where $u=1 / r, G M$ is the heliocentric gravitational constant, $M$ is the mass of the Sun, $J=r^{2} \dot{\theta}=\mathrm{rv}=\mathrm{v} / \mathrm{u}$ 。

$$
\frac{d^{2} \mathrm{u}}{d \theta^{2}}+\mathrm{u}=\frac{G M}{J^{2}}=\frac{F}{m \mathrm{u}^{2} J^{2}}
$$

We get the necessary data from the NASA official website and display it in Table2 and Table3.

Table2 parameters of Planets ${ }^{7}$

\begin{tabular}{|l|r|c|r|r|}
\hline \multicolumn{1}{|c|}{ parameters } & Mercury & Venus & \multicolumn{1}{c|}{ Earth } & \multicolumn{1}{l|}{ Mars } \\
\hline Mass $\left(10^{24} \mathrm{~kg}\right)$ & 0.33011 & 4.8675 & 5.9723 & 0.64171 \\
\hline Radius $\mathrm{R}_{\mathrm{m}}\left(10^{6} \mathrm{~m}\right)$ & 2.4397 & 6.0518 & 6.371 & 3.3895 \\
\hline perihelion $\mathrm{r}_{\min }\left(10^{9} \mathrm{~m}\right)$ & 46.00 & 107.48 & 147.09 & 206.62 \\
\hline aphelion $\mathrm{r}_{\max }\left(10^{9} \mathrm{~m}\right)$ & 69.82 & 108.94 & 152.10 & 249.23 \\
\hline Period $($ day $)$ & 87.969 & 224.701 & 365.256 & 686.980 \\
\hline velocity $\overline{\mathrm{v}}(\mathrm{m} / \mathrm{s})$ & 47360 & 35020 & 29780 & 24070 \\
\hline $\mathrm{J}\left(10^{15} \mathrm{~m}^{2} / \mathrm{s}\right)$ & 2.712981 & 3.789459 & 4.455097 & 5.475773 \\
\hline
\end{tabular}

Table 3 parameters of Sun $^{8}$

\begin{tabular}{|l|c|}
\hline \multicolumn{1}{|c|}{ parameters } & values \\
\hline Mass $\left(10^{30} \mathrm{~kg}\right)$ & 1.9885 \\
\hline $\mathrm{GM}\left(10^{20} \mathrm{~m}^{3} / \mathrm{s}^{2}\right)$ & 1.32712 \\
\hline Radius $\mathrm{R}_{\mathrm{M}}\left(10^{8} \mathrm{~m}\right)$ & 6.957 \\
\hline
\end{tabular}

All planets in the solar system meet the condition $\mathrm{m} / 2>$ $\mathrm{MS}_{\mathrm{m}} / \mathrm{S}$, the correction coefficient $\mathrm{K}$ of gravitation can be calculate using the Eq.(10) (11) (12), and because $r>>R_{m}$ and $r>R_{M}$ it can be simplified to the form of Eq.(20) by using curve fitting, the fitting results are shown in Table4.

$$
K=\frac{\sqrt{S_{M} S_{m}}}{S_{r}} \approx \mathrm{Au}^{2}
$$

Table4 results of curve fitting of Eq.(20)

\begin{tabular}{|c|c|c|}
\hline Planets & the value of A & Absolute error of K \\
\hline Mercury & $4.243335 \mathrm{E} 14$ & $\pm 1.6 \mathrm{E}-12$ \\
\hline Venus & $1.05256477 \mathrm{E} 15$ & $\pm 7.1 \mathrm{E}-15$ \\
\hline Earth & $1.10807918 \mathrm{E} 15$ & $\pm 5.6 \mathrm{E}-15$ \\
\hline Mars & $5.8951947 \mathrm{E} 14$ & $\pm 6.3 \mathrm{E}-15$ \\
\hline
\end{tabular}

An arcsec is about 7.716E-07 of one circumference, so the absolute error of $\mathrm{K}$ in Table4 is small enough, and then the Eq.(9) approximate becomes:

$$
F=\left(1+\mathrm{Au}^{2}\right) G M m \mathrm{u}^{2}
$$

From Eq. (19) and Eq.(21) we obtain:

$$
\frac{d^{2} \mathrm{u}}{d \theta^{2}}+\mathrm{u}=\frac{G M}{J^{2}}\left(1+\mathrm{Au}^{2}\right)
$$

And then:

$$
\frac{d^{2} \mathrm{u}}{d \theta^{2}}+\mathrm{u}\left(1-\frac{\mathrm{GMAu}}{J^{2}}\right)=\frac{G M}{J^{2}}
$$

Eq.(23) can be approximately regarded as a differential equation of simple harmonic motion, the contents in parentheses of Eq.(23) is the square of the orbital angular frequency of the planet, then:

$$
\omega=\sqrt{1-\frac{\mathrm{GMAu}}{J^{2}}}
$$


The orbit angular frequency $\omega$ in Eq.(24) is a variable that depends on $u$, so the $\omega$ needs to be averaged, and it is more reasonable to use orbital average velocity $\bar{v}$ instead of $\bar{u}$ to find $\bar{\omega}$ (the average value of $\omega$ ), and because $u=v / J$, then:

$$
\bar{\omega}=\sqrt{1-\frac{\mathrm{GMA} \overline{\mathrm{V}}}{J^{3}}}
$$

The precession value in one orbit period is:

$$
\Delta \emptyset=360 \times 3600 \times\left(\frac{1}{\bar{\omega}}-1\right)
$$

Table 5 the precession of perihelion of planets in a century

\begin{tabular}{|c|c|c|c|}
\hline Planets & $\begin{array}{c}\text { observed }^{9} \\
\text { "/cy }\end{array}$ & $\begin{array}{c}\text { GTR }^{9} \\
\text { "/cy }\end{array}$ & $\begin{array}{c}\Delta \emptyset_{1 c y} \\
\text { "/cy }\end{array}$ \\
\hline Mercury & $\begin{array}{l}\text { Le Verrier: } 38 \\
\text { Newcomb:43.11 } \pm 0.45\end{array}$ & 43.5 & 35.94 \\
\hline Venus & $8.4 \pm 4.8$ & 8.62 & 9.47 \\
\hline Earth & $5.0 \pm 1.2$ & 3.87 & 3.21 \\
\hline Mars & $1.3624 \pm 0.0005$ & 1.36 & 0.395 \\
\hline
\end{tabular}

\section{ANALYSIS AND DISCUSSION}

Our error correction formulas significantly reduced the abnormal precession of Mercury: The total observed precession of Mercury is $5600 " / \mathrm{cy}^{11}$, the abnormal precession calculated by Newcomb is about $43 " / \mathrm{cy}^{9}$, and the error $=43 / 5600=7.68 \%$, the abnormal precession calculated by Le Verrier is $38 \mathrm{H} / \mathrm{cy}^{9}$, the error is $6.79 \%$, our new theory contributes $35.94 " / \mathrm{cy}$, compared with Newcomb's data, the abnormal precession reduce to about $7 " /$ cy with an error $1.25 \%$, compared with the data of Le Verrier, the abnormal precession reduce to about 2"/cy with error $0.357 \%$.

Many papers question that the abnormal precession of mercury perihelion equals $43 \mathrm{H} / \mathrm{cy}$. Einstein use formula (27) for calculating the abnormal precession of Mercury, in fact, Einstein had made several approximate treatments when he got formula (27) ${ }^{12,13,14}$.

$$
\varepsilon=\frac{24 \pi^{3} a^{2}}{\left(1-e^{2}\right) c^{2} T^{2}}
$$

Hua Di's paper shows that Einstein was wrong in integral calculation, the abnormal precession of Mercury calculate by Einstein's GTR should be $71.5 " / \mathrm{cy}^{12}$, and Kupryaev agrees with Hua Di's viewpoint, his paper shows a result $71.63 " /$ cy ${ }^{13}$. However, the maximum absolute error of $\mathrm{K}$ caused by the approximate treatment of Eq. (20) in this paper is only about $1.6 \mathrm{e}-12$ (equivalent to $0.00086 \mathrm{k} / \mathrm{cy}$ ).

The mass ring model used by Newcomb and Le Verrier in the calculation of the perturbation of the planets to Mercury had made approximate treatment too. Price and Rush shows the calculating process by the mass ring model in their paper in 1979. ${ }^{10}$ This model only considers the influence of radial direction and does not consider other directions, and all planets actually revolve around the center of mass of the solar system, not around the sun, for example, the common center of mass of Jupiter and the sun is located at about 1.07 times of the radius of the sun, that is to say, outside the surface of the sun. So, we don't think the observed value of the precession of Mercury is a fixed value, it changes over time, Rydin also shows a similar viewpoint in $2011^{11}$

So, 43"/cy should not be the evidence for prove that Einstein's GTR is correct. The 35.94"/cy is not the exact abnormal precession value too, our formulas just only contributes about 36"/cy to theoretical calculation value of mercury precession. Obviously, measurement errors always exist, no one can eliminate it completely. If convert the remaining abnormal precession 2"/cy to the measurement error of Mercury's parameters, it will be incredibly accurate. So, Eq. (16) in this paper is reliable.

And it is not a coincidence that some of the measured $G$ values by some famous surveyors using different devices at different positions coincide with $1 /(16 \pi \mathrm{c})$.

We regard the gravitational field as flow field of ideal fluid, the gravitation between tangible masses is not a strict inverse square relation when the distance is very close, which needs to be corrected. For tangible mass, the simplest case is that both masses are uniform spheres, just like Cavendish's experiment. So, we consider the following principles when we choose the experimental data of G measurement to verify Eq. (7) (15) (16) : the large mass must be sphere, and only two large masses per measurement, and the surveyors provides all dimensions of the large and the small masses and the distance between the centers of the large masse and the small masse. In fact, most of the surveyors did not use uniform spheres for the large mass and the small mass at the same time in their experimenters, this will cause the gravitational field to become complex.

If the gravitational field can be regard as flow field of ideal fluid, in addition to the correction method we mentioned, there are other factors may affect the measurement accuracy of $\mathrm{G}$ values. For example, modern equipment for measuring $\mathrm{G}$ value is very compact, the distance between the supporting parts of the torsion balance and the large ball and the small ball is very small, it may interfere with the gravitational field. And the test mass is too small, for example, the two small masses used by Luther is only 5 grams ${ }^{5,6}$, the influence of the supporting parts of the torsion balance on the gravitational field is much greater than the influence of the small mass. Thus, there may be an illusion, the accuracy of the measured $\mathrm{G}$ value is very high for one team. But the measured $\mathrm{G}$ value do not coincide with other teams as the equipment used is not the same.

We gave up some experimental data, here are some examples:

Boys only provided the centroid distance between the large ball and the small ball of the Exp8 in his paper in $1895^{15}$. We can only assume the dimension of $\mathrm{p}$ or $\mathrm{b}$ (in Fig. E) ${ }^{15}$ invariant to correct the measured $\mathrm{G}$ value of other experiments, and the relative errors between the results of boys are very large, three of which are more than 2000ppm.

Luther used the same large mass $\mathrm{M}$ in 1997 as the experiments in $1982^{6}$, the diameter of the large mass $\mathrm{M}$ is about $101.65 \mathrm{~mm}^{5}$, but his paper in 1997 shows that the separation of the centers of the tungsten spheres (large mass) is $71.9092 \mathrm{~mm}$ for Expt.\#1 and $69.8567 \mathrm{~mm}$ for Expt.\#2 ${ }^{6}$, less than $101.65 \mathrm{~mm}$, this is impossible, and the determination employing low-Q torsion pendulums have an upward bias, so we think the experimental data of Luther in 1997 may had problems and did not use his data in paper 1997.

Our theory given a completely different explanation from Einstein's GTR for the abnormal precession of Mercury, and only one of the explanations is right. We designed an experiment for the space agency to further verify which is right:

Launch a spacecraft that rotates around the sun which orbit 
parameters is close to the Mercury's orbit, is about 180 degrees of phase difference. It can verify whether Einstein's GTR is correct or our theory is correct by measuring the orbit precession of the spacecraft. Consider Kepler's law, we can obtain Eq. (28) from formula (27):

$$
\varepsilon=\frac{3 G M}{c^{2} R_{p}} \cdot \frac{2-e}{\left(1-e^{2}\right)}=\frac{3 G M}{c^{2} R_{p}} \cdot f(e)
$$

We using Eq.(28) for qualitative analysis, not for accurate calculation, it can be approximately considered that $\mathrm{M}$ is the mass of the sun, $R_{p}$ is the distance between the sun and the perihelion, and $\mathrm{e}$ is the eccentricity of the orbit. According to Eq. (28), if the perihelion of the spacecraft is kept constant, the orbit precession of the spacecraft will only change with the eccentricity of the orbit. And because the spacecraft is very small, according to Eq. (16), $\mathrm{K}_{\mathrm{m}}$ is very small too, and then the calculated orbit precession value of the spacecraft is close to zero. So, if the measured orbit precession value of the spacecraft is close to that of Mercury, Einstein is right, if the measured orbit precession value of the spacecraft is close to zero (no precession), our theory is right. Change the orbit and let the eccentricity e to be a large value, and the perihelion kept constant, according to Eq.(28), the orbit precession value should change a lot, according to our theory, the orbit precession value of the spacecraft change too, but still very close to zero.

Compared with Einstein's theory of relativity, our theory only uses elementary mathematics, each variable and coefficient in the equations has a specific physical meaning, and has the characteristics of simple beauty, and the correction method can be applied in a wider range, while Einstein's GTR does not explain why the $G$ value measured by different teams do not coincide.

\section{CONCLUSIONS}

1.The exact value of gravitational constant can be derived from the speed of light in vacuum, $\mathrm{G}=1 /(16 \pi \mathrm{c})$.

2.If two masses are tangible and very close, the gravitation and centroid distance is not strictly inverse square relation.

3.The gravitation in the measurement of $\mathrm{G}$ value by torsion balance needs to be corrected.

4.The abnormal precession of Mercury equals 43"/cy should not be the evidence for prove that Einstein's GTR is correct.

\section{ACKNOWLEDGMENTS}

We have no conditions for related experiments, our research all depends on the previous experimental data, thanks all the scientists for their research and their experimental data.

\section{REFERENCES}

1. Mohr, J. P., Newell, B. D. \& Taylor, N. B. CODATA Recommended Values of the Fundamental Physical Constants: 2014*. J. Phys. Chem. Ref. Data 45, 043102(2016).

2. Li, Q., Xue, C. \& Liu, J., P., et al. Measurements of the gravitational constant using two independent methods. Nature 506, 582588(2018).

3. Liu, J. P. Determination of the Newtonian gravitational constant $\mathrm{G}$ with time-of-swing method using high-Q silica fibers. $\mathrm{PhD}$ thesis (Huazhong University of Science \& Technology, China, 2019); http://kns.cnki.net/KCMS/detail/detail.aspx?dbcode=CDFD\&filen ame $=1019600696 . n h$.
4. Cavendish, H. Experiments to determine the density of the Earth Phil. Trans. R. Soc. London 88, 469-526(1798).

5. Luther, G. G. \& Towler, R. W. Redetermination of the Newtonian Gravitational Constant G. Phys. Rev. Lett. 48, 121-123(1982).

6. Bagley, H. C. \& Luther, G. G. Preliminary Results of a Determination of the Newtonian Constant of Gravitation: A Test of the Kuroda Hypothesis. Phys. Rev. Lett. 78, 3047-3050(1997).

7. Williams, D. R. (21 October 2019). Planetary Fact Sheet - Metric. NASA. https://nssdc.gsfc.nasa.gov/planetary/factsheet/.

8. Williams, D. R. (23 February 2018). Sun Fact Sheet. NASA. https://nssdc.gsfc.nasa.gov/planetary/factsheet/sunfact.html.

9. Nyambuya, G. G. Azimuthally symmetric theory of gravitation - I. On the perihelion precession of planetary orbits. Mon. Not. R. Astron. Soc. 403, 1381-1391(2010).

10. Price, M. \& Rush, W. Nonrelativistic contribution to Mercury's perihelion precession. Am. J. Phys. 47, 531-534 (1979)

11. Rydin, A. R. The Theory of Mercury's Anomalous Precession (Proceedings of the NPA Vol.8, College Park, MD 2011).

12. Hua, Di. Einstein's Explanation of Perihelion Motion of Mercury. In Smarandache, F., Fu, Y. H. \& Zhao, F. J. (Eds.), Unsolved Problems in Special and General Relativity (pp.3-7). Education Publishing, Columbus, 2013.

13. Kupryaev, N. V. Concerning the paper by A. Einstein "Explanation of the Perihelion Motion of Mercury from the General Theory of Relativity". Russian Physics Journal 61, 648-653(2018)

14. Einstein A. Erklärung der Perihelbewegung des Merkur aus der allgemeinen Realtivitätstheorie (Sitzungsberichte der Preussischen Akademie der Wissenschaften 831-839, Berlin, 1915)

15. Boys, C. V. On the Newtonian Constant of Gravitation. Philos. Trans. R. Soc. London 186, 1-72(1895) 